\title{
Discrete Integrable Equations over Finite Fields
}

\author{
Masataka KANKI ${ }^{\dagger}$, Jun MADA ${ }^{\ddagger}$ and Tetsuji TOKIHIRO ${ }^{\dagger}$ \\ $\dagger$ Graduate School of Mathematical Sciences, University of Tokyo, \\ 3-8-1 Komaba, Tokyo 153-8914, Japan \\ E-mail: kanki@ms.u-tokyo.ac.jp,toki@ms.u-tokyo.ac.jp \\ $¥$ College of Industrial Technology, Nihon University, \\ 2-11-1 Shin-ei, Narashino, Chiba 275-8576, Japan \\ E-mail: mada.jun@nihon-u.ac.jp
}

Received May 18, 2012, in final form August 15, 2012; Published online August 18, 2012 http://dx.doi.org/10.3842/SIGMA.2012.054

\begin{abstract}
Discrete integrable equations over finite fields are investigated. The indeterminacy of the equation is resolved by treating it over a field of rational functions instead of the finite field itself. The main discussion concerns a generalized discrete KdV equation related to a Yang-Baxter map. Explicit forms of soliton solutions and their periods over finite fields are obtained. Relation to the singularity confinement method is also discussed.
\end{abstract}

Key words: integrable system; discrete KdV equation; finite field; cellular automaton

2010 Mathematics Subject Classification: 35Q53; 37K40; 37P25

\section{Introduction}

Cellular automata are discrete dynamical systems which provide simple and efficient tools for modeling complex phenomena [17]. Since each cell of a cellular automaton takes only a finite number of states, it seems natural to describe its time evolution by utilising a finite field. In particular, if we can construct finite field analogues of dynamical equations whose mathematical structures are well studied, such as integrable systems, this construction may give some fundamental methods for analysing models of cellular automata. Discrete analogues of integrable equations have been widely investigated, however, their extension over a finite field has less been examined. One of the reasons for this may be that the time evolution of a nonlinear system is not always well defined over a finite field. For example, let us consider the discrete $\mathrm{KdV}$ equation

$$
\frac{1}{x_{n+1}^{t+1}}-\frac{1}{x_{n}^{t}}+\frac{\delta}{1+\delta}\left(x_{n}^{t+1}-x_{n+1}^{t}\right)=0
$$

over a finite field $\mathbb{F}_{q}$ where $q=p^{m}, p$ is a prime number and $m \in \mathbb{Z}_{+}$. Here $n, t \in \mathbb{Z}$ and $\delta$ is a parameter. If we put

$$
\frac{1}{y_{n}^{t}}:=(1+\delta) \frac{1}{x_{n}^{t+1}}-\delta x_{n}^{t}
$$

we obtain equivalent coupled equations

$$
x_{n}^{t+1}=\frac{(1+\delta) y_{n}^{t}}{1+\delta x_{n}^{t} y_{n}^{t}}, \quad y_{n+1}^{t}=\frac{\left(1+\delta x_{n}^{t} y_{n}^{t}\right) x_{n}^{t}}{1+\delta} .
$$

Clearly (1.2) does not determine the time evolution when $1+\delta x_{n}^{t} y_{n}^{t} \equiv 0$. Over a field of characteristic 0 such as $\mathbb{C}$, the time evolution of $\left(x_{n}^{t}, y_{n}^{t}\right)$ will not hit this exceptional line for 
generic initial conditions, but on the contrary, the evolution comes to this exceptional line in many cases over a finite field as a division by 0 appears.

A pioneering work on integrable equations over finite fields is that by Doliwa, Białecki and Klimczewski [1,4]. They used an algebro-geometric approach to construct soliton solutions to discrete integrable equations over finite fields in Hirota's bilinear form. For the discrete $\mathrm{KdV}$ equation, the bilinear form is written as

$$
(1+\delta) \sigma_{n+1}^{t+1} \sigma_{n}^{t-1}=\delta \sigma_{n+1}^{t-1} \sigma_{n}^{t-1}+\sigma_{n}^{t} \sigma_{n+1}^{t} .
$$

The $N$-soliton solution to the equation (1.3) is given as

$$
\sigma_{n}^{t}=\operatorname{det}_{1 \leq i, j \leq N}\left(\delta_{i j}+\frac{\gamma_{i}}{l_{i}+l_{j}-1}\left(\frac{1-l_{i}}{l_{i}}\right)^{t}\left(\frac{l_{i}+\delta}{1+\delta-l_{i}}\right)^{n}\right)
$$

where $\gamma_{i}, l_{i}(i=1,2, \ldots, N)$ are arbitrary parameters satisfying $l_{i} \neq l_{j}$ for $i \neq j$. Hence if we choose $l_{i} \not \equiv 0,1+\delta(i=1,2, \ldots, N)$ and $l_{i}+l_{j} \not \equiv 1(1 \leq i, j \leq N)$, the $N$ soliton solution (1.4) is well defined for all $(n, t) \in \mathbb{Z}^{2}$ and gives a time evolution pattern over a finite field. A similar approach was also used for discrete KP equation in the bilinear form [2]. However, since the nonlinear form of the discrete $\mathrm{KdV}$ equation (1.1) is obtained from (1.3) by

putting $x_{n}^{t}:=\frac{\sigma_{n}^{t} \sigma_{n+1}^{t-1}}{\sigma_{n+1}^{t} \sigma_{n}^{t-1}}$, well defined $N$-soliton solutions $x_{n}^{t}$ of (1.1) or (1.2) cannot be obtained from (1.4) because $x_{n}^{t}$ is not defined if $\sigma_{n+1}^{t} \equiv 0$ or $\sigma_{n}^{t-1} \equiv 0$. Indeterminacy of the time evolution for a generic initial state cannot be avoided either when we use Hirota's bilinear form. Note that if we consider the equation over $\mathbb{P F}_{q}:=\mathbb{F}_{q} \cup\{\infty\}$ instead of $\mathbb{F}_{q}$ by adding a value $\infty$, we frequently hit the indeterminate values $\frac{0}{0}, \infty+0,0 \cdot \infty$ and so on, which causes further problems.

In this article, we propose a prescription to determine the time evolution of a nonlinear system over finite fields by taking as examples the discrete $\mathrm{KdV}$ equation (1.1) and its generalization. We show in Section 2 that the initial value problem is well defined and we investigate $N$-soliton solutions in Section 3. The last section is devoted to discussing the relation of our method to the singularity confinement method [5].

\section{A generalized discrete $\mathrm{KdV}$ equation over a function field}

\section{$2.1 \quad$ Discrete $\mathrm{KdV}$ equation}

First we explain how the indeterminate values appear through the time evolution by examining the discrete $\mathrm{KdV}$ equation (1.2) over $\mathbb{F}_{7}:=\{0,1,2,3,4,5,6\}$. If we take $\delta=1$, (1.2) turns into

$$
x_{n}^{t+1}=\frac{2 y_{n}^{t}}{1+x_{n}^{t} y_{n}^{t}}, \quad y_{n+1}^{t}=\frac{\left(1+x_{n}^{t} y_{n}^{t}\right) x_{n}^{t}}{2} .
$$

Suppose that $x_{1}^{0}=6, x_{2}^{0}=5, y_{1}^{0}=2, y_{1}^{1}=2$, then we have

$$
x_{1}^{1}=\frac{4}{13} \equiv 3, \quad y_{2}^{0}=\frac{78}{2} \equiv 4 \quad \bmod 7 .
$$

With further calculation we have

$$
x_{1}^{2}=\frac{4}{7} \equiv \frac{4}{0}, \quad y_{2}^{1}=\frac{21}{2} \equiv 0, \quad x_{2}^{1}=\frac{8}{21} \equiv \frac{1}{0} .
$$

Since $\frac{4}{0}$ and $\frac{1}{0}$ are not defined over $\mathbb{F}_{7}$, we now extend $\mathbb{F}_{7}$ to $\mathbb{P F}_{7}$ and take $\frac{j}{0} \equiv \infty$ for $j \in$ $\{1,2,3,4,5,6\}$. However, at the next time step, we have

$$
x_{2}^{2}=\frac{2 \cdot 0}{1+\infty \cdot 0}, \quad y_{3}^{1}=\frac{(1+\infty \cdot 0) \cdot \infty}{2}
$$

and reach a deadlock. 


$$
\begin{aligned}
& \begin{array}{lll}
x_{1}^{0}=6 & x_{2}^{0}=5 & x_{3}^{0}=4
\end{array} \\
& y_{1}^{0}=2 \\
& x_{1}^{1}=3 \quad x_{2}^{1}=\infty \quad x_{3}^{1}=0 \\
& y_{1}^{1}=2 \quad y_{2}^{1}=0 \quad y_{3}^{1}=5 \\
& x_{1}^{2}=\infty \quad x_{2}^{2}=4
\end{aligned}
$$

Figure 1. An example of the time evolution of the coupled discrete KdV equation (1.2) over $\mathbb{P F}_{7}$ where $\delta=1$.
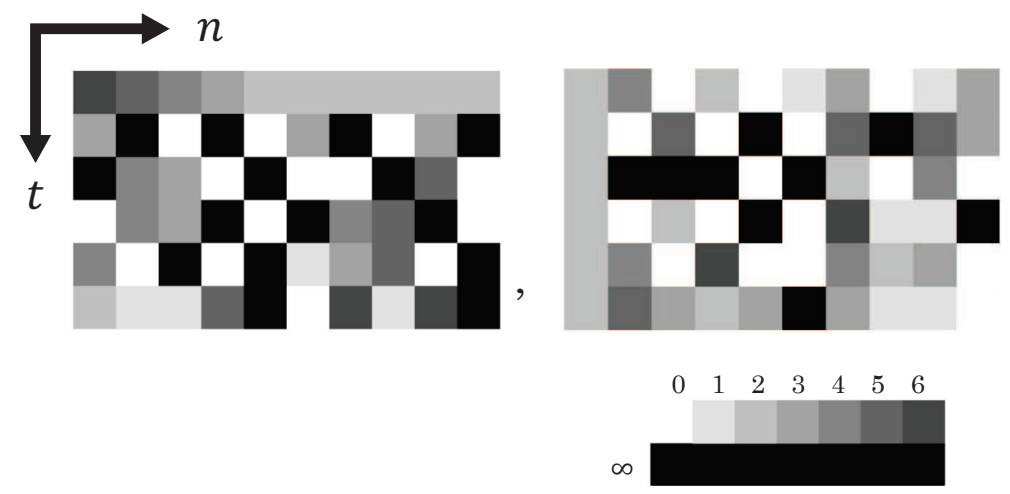

Figure 2. The time evolution pattern of $x_{n}^{t}$ (left) and $y_{n}^{t}$ (right) of (1.2) over $\mathbb{P F}_{7}$ where $\delta=1$. Elements of $\mathbb{P F}_{7}$ are represented on the following grayscale: from 0 (white) to 6 (gray) and $\infty$ (black). See the scale bar in the figure.

Therefore we try the following two procedures: [I] we keep $\delta$ as a parameter for the same initial condition, and obtain as a system over $\mathbb{F}_{7}(\delta)$,

$$
\begin{aligned}
& x_{1}^{1}=\frac{2(1+\delta)}{1+5 \delta}, \quad y_{2}^{0}=\frac{6(1+5 \delta)}{1+\delta}, \quad x_{2}^{1}=\frac{6(1+\delta)(1+5 \delta)}{1+3 \delta+3 \delta^{2}}, \quad y_{2}^{1}=\frac{2\left(1+2 \delta+4 \delta^{2}\right)}{(1+5 \delta)^{2}}, \\
& x_{1}^{2}=\frac{2(1+\delta)(1+5 \delta)}{1+2 \delta+4 \delta^{2}}, \quad x_{2}^{2}=\frac{4(1+\delta)(2+\delta)(3+2 \delta)}{(1+5 \delta)\left(5+5 \delta+2 \delta^{2}\right)}, \quad y_{3}^{1}=\frac{2\left(5+5 \delta+2 \delta^{2}\right)}{(2+\delta)^{2}} .
\end{aligned}
$$

[II] Then we put $\delta=1$ to have a system over $\mathbb{P F}_{7}$ as

$$
\begin{aligned}
& x_{1}^{1}=3, \quad y_{2}^{0}=4, \quad x_{2}^{1}=\frac{72}{7} \equiv \infty, \quad y_{2}^{1}=\frac{14}{36} \equiv 0, \quad x_{1}^{2}=\frac{24}{7} \equiv \infty, \\
& x_{2}^{2}=\frac{120}{72} \equiv 4, \quad y_{3}^{1}=\frac{24}{9} \equiv 5 .
\end{aligned}
$$

Thus all the values are uniquely determined over $\mathbb{P F}_{7}$. Figs. 1 and 2 show a time evolution pattern of the discrete $\mathrm{KdV}$ equation (1.2) over $\mathbb{P F}_{7}$ for the initial conditions $x_{1}^{0}=6, x_{2}^{0}=5$, $x_{3}^{0}=4, x_{4}^{0}=3, x_{j}^{0}=2(j \geq 5)$ and $y_{1}^{t}=2(t \geq 0)$.

This example suggests that the equation (1.2) should be understood as evolving over the field $\mathbb{F}_{q}(\delta)$, the rational function field with indeterminate $\delta$ over $\mathbb{F}_{q}$. To obtain the time evolution pattern over $\mathbb{P F}_{q}$, we have to substitute $\delta$ with a suitable value $\delta_{0} \in \mathbb{F}_{q}\left(\delta_{0}=1\right.$ in the example 
above). This substitution can be expressed as the following reduction map:

$$
\mathbb{F}_{q}(\delta)^{\times} \rightarrow \mathbb{P F}_{q}:\left(\delta-\delta_{0}\right)^{s} \frac{g\left(\delta-\delta_{0}\right)}{f\left(\delta-\delta_{0}\right)} \mapsto \begin{cases}0, & s>0, \\ \infty, & s<0 \\ \frac{g(0)}{f(0)}, & s=0\end{cases}
$$

where $s \in \mathbb{Z}, f(h), g(h) \in \mathbb{F}_{q}[h]$ are co-prime polynomials and $f(0) \neq 0, g(0) \neq 0$. With this prescription, we know that $0 / 0$ does not appear and we can uniquely determine the time evolution for generic initial conditions defined over $\mathbb{F}_{q}$.

\subsection{Generalized discrete $\mathrm{KdV}$ equation}

In this subsection we explain how to apply our method to a dynamical system with more than one parameters by taking a generalized form of the discrete $\mathrm{KdV}$ equation as an example. In this case, we have to be careful in substituting the values to the parameters. The generalised discrete $\mathrm{KdV}$ equation is the following discrete integrable system:

$$
x_{n}^{t+1}=\frac{\left\{(1-\beta)+\beta x_{n}^{t} y_{n}^{t}\right\} y_{n}^{t}}{(1-\alpha)+\alpha x_{n}^{t} y_{n}^{t}}, \quad y_{n+1}^{t}=\frac{\left\{(1-\alpha)+\alpha x_{n}^{t} y_{n}^{t}\right\} x_{n}^{t}}{(1-\beta)+\beta x_{n}^{t} y_{n}^{t}},
$$

with arbitrary parameters $\alpha$ and $\beta$. To avoid indeterminacy, we regard (2.1) as a dynamical system over $\mathbb{F}_{q}(\alpha, \beta)$. Then, as in the case of (1.2), its time evolution is uniquely determined for generic initial and boundary conditions. Note that when we substitute values in $\mathbb{F}_{q}$ for the parameters, the result can be indeterminate, i.e., $\frac{0}{0}$, or it can depend on the order of the substitutions. These problems are typical of a field of rational functions with two or more parameters. Even if the numerator and the denominator of a rational function are both irreducible polynomials without common factors, there will be points of indeterminacy, since the numerator and the denominator intersect in co-dimension two or more in the space of parameters. For example, let $q=5$ and suppose that $x_{n}^{t}=y_{n}^{t}=2 \in \mathbb{F}_{5}$ then

$$
x_{n}^{t+1}=\frac{2+\beta}{1+3 \alpha} \in \mathbb{F}_{5}(\alpha, \beta) .
$$

If we put $\alpha=\beta=3 \in \mathbb{F}_{5}$, then, we find that

$$
x_{n}^{t+1}=\frac{0}{0},
$$

or, if we first substitute $\beta$, then

$$
x_{n}^{t+1}=\frac{0}{1+3 \alpha} \equiv 0,
$$

which is unrelated to subsequent substitutions of $\alpha$.

One remedy is to regard these parameters themselves as depending on a common parameter. For example, if we put $\alpha=3+\epsilon$ and $\beta=3+\epsilon$,

$$
x_{n}^{t+1}=\frac{2+(3+\epsilon)}{1+3(3+\epsilon)} \equiv \frac{\epsilon}{3 \epsilon}=\frac{1}{3} \equiv 2,
$$

and the value is uniquely determined in $\mathbb{P F}_{5}$. We show an example of a time evolution pattern of (2.1) thus determined in Fig. 3. The preceding arguments suggest a general trick to construct an equation, or a time evolution rule, over a finite field from a given discrete equation. 


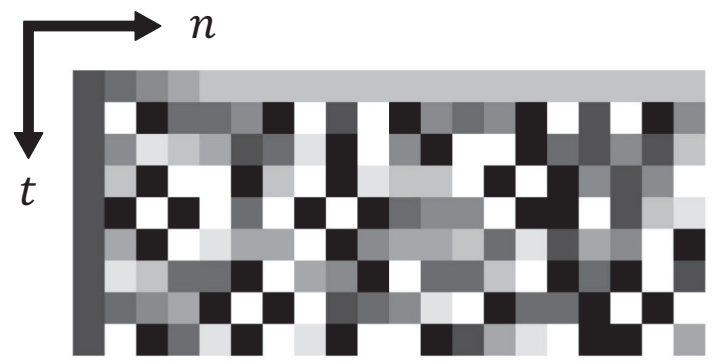

Figure 3. The time evolution pattern of $x_{n}^{t}$ of the generalised discrete KdV equation (2.1) over $\mathbb{P F}_{7}$ where $\alpha=2, \beta=3$. Elements of $\mathbb{P F}_{7}$ are represented on the following grayscale: from 0 (white) to 6 (gray) and $\infty$ (black).

1. Introduce one parameter, say $\epsilon$, in the equation (or the initial condition), and obtain a solution over $\mathbb{F}_{q}(\epsilon)$.

2. Substitute a value in $\mathbb{F}_{q}$ for the parameter in the solution, and obtain a pattern over $\mathbb{P F}_{q}$.

This construction can be applied to both ordinary and partial difference equations regardless of their integrability. If we have explicit form of a solution with this parameter, we immediately obtain a pattern over a finite field by replacing the parameter with a value in the field. In the next section, we show some example of soliton solutions of (1.2) and (2.1) over finite fields.

\section{Soliton solutions over finite fields}

First we consider the $N$-soliton solutions to (1.1) over $\mathbb{F}_{q}$. As mentioned in the introduction, the $N$-soliton solution is given as

$$
x_{n}^{t}=\frac{\sigma_{n}^{t} \sigma_{n+1}^{t-1}}{\sigma_{n+1}^{t} \sigma_{n}^{t-1}}, \quad \sigma_{n}^{t}:=\operatorname{det}_{1 \leq i, j \leq N}\left(\delta_{i j}+\frac{\gamma_{i}}{l_{i}+l_{j}-1}\left(\frac{1-l_{i}}{l_{i}}\right)^{t}\left(\frac{l_{i}+\delta}{1+\delta-l_{i}}\right)^{n}\right),
$$

where $\gamma_{i}, l_{i}(i=1,2, \ldots, N)$ are arbitrary parameters but $l_{i} \neq l_{j}$ for $i \neq j$. When $l_{i}, \gamma_{i}$ are chosen in $\mathbb{F}_{q}, x_{n}^{t}$ becomes a rational function in $\mathbb{F}_{q}(\delta)$. Hence we obtain soliton solutions over $\mathbb{P F}_{q}$ by substituting $\delta$ with a value in $\mathbb{F}_{q}$. Figs. 4 and 5 show one and two soliton solutions for the discrete $\mathrm{KdV}$ equation (1.1) over the finite fields $\mathbb{P F}_{11}$ and $\mathbb{P F}_{19}$. The corresponding time evolutionary patterns on the field $\mathbb{R}$ are also presented for comparison.

Next we consider soliton solutions to the generalized discrete KdV equation (2.1). Note that by putting $u_{n}^{t}:=\alpha x_{n}^{t}, v_{n}^{t}:=\beta y_{n}^{t}$, we obtain

$$
u_{n}^{t+1}=\frac{\left(\alpha(1-\beta)+u_{n}^{t} v_{n}^{t}\right) v_{n}^{t}}{\beta(1-\alpha)+u_{n}^{t} v_{n}^{t}}, \quad v_{n+1}^{t}=\frac{\left(\beta(1-\alpha)+u_{n}^{t} v_{n}^{t}\right) u_{n}^{t}}{\alpha(1-\beta)+u_{n}^{t} v_{n}^{t}} .
$$

Hence (2.1) is essentially equivalent to the 'consistency of the discrete potential KdV equation around a 3 -cube' [12]: $(u, v) \rightarrow\left(u^{\prime}, v^{\prime}\right)$, as

$$
u^{\prime}=v P, \quad v^{\prime}=u P^{-1}, \quad P=\frac{a+u v}{b+u v} .
$$

The map is also obtained from discrete BKP equation [6]. We will obtain $N$-soliton solutions to (2.1) from the $N$-soliton solutions to the discrete KP equation by a reduction similar to the one adopted in $[6]$. 


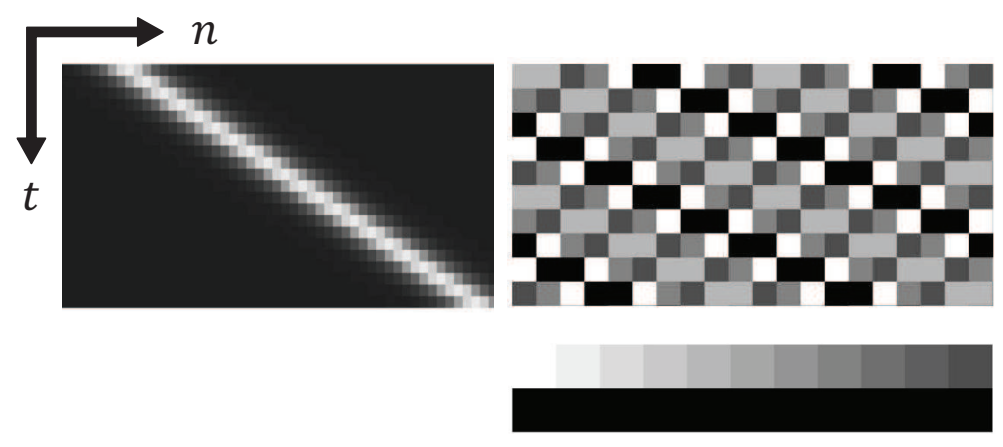

Figure 4. The one-soliton solution of the discrete $\mathrm{KdV}$ equation (1.1) over $\mathbb{R}$ (left) and $\mathbb{P F}_{11}$ (right) where $\delta=7, \gamma_{1}=2, l_{1}=9$. Elements of $\mathbb{P F}_{11}$ are represented on the following grayscale: from 0 (white) to 10 (gray) and $\infty$ (black).
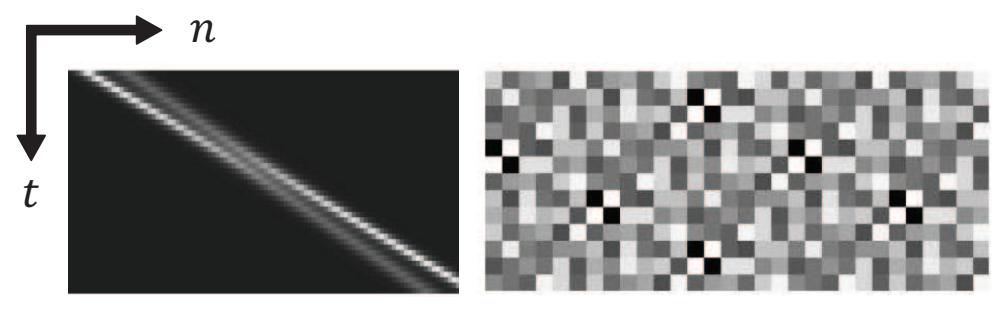

Figure 5. The two-soliton solution of the discrete $\mathrm{KdV}$ equation (1.1) over $\mathbb{R}$ (left) and $\mathbb{P F}_{19}$ (right) where $\delta=8, \gamma_{1}=15, l_{1}=2, \gamma_{2}=9, l_{2}=4$. Elements of $\mathbb{P F}_{19}$ are represented on the following grayscale: from 0 (white) to 18 (gray) and $\infty$ (black). It is difficult to see the interaction of solitons over $\mathbb{P F}_{19}$.

Let us consider the four-component discrete KP equation:

$$
\begin{aligned}
& \left(a_{1}-b\right) \tau_{l_{1} t} \tau_{n}+(b-c) \tau_{l_{1}} \tau_{t n}+\left(c-a_{1}\right) \tau_{l_{1} n} \tau_{t}=0, \\
& \left(a_{2}-b\right) \tau_{l_{2} t} \tau_{n}+(b-c) \tau_{l_{2}} \tau_{t n}+\left(c-a_{2}\right) \tau_{l_{2} n} \tau_{t}=0 .
\end{aligned}
$$

Here $\tau=\tau\left(l_{1}, l_{2}, t, n\right)\left(\left(l_{1}, l_{2}, t, n\right) \in \mathbb{Z}^{4}\right)$ is the $\tau$-function, and $a_{1}, a_{2}, b, c$ are arbitrary parameters and we use the abbreviated form, $\tau \equiv \tau\left(l_{1}, l_{2}, t, n\right), \tau_{l_{1}} \equiv \tau\left(l_{1}+1, l_{2}, t, n\right), \tau_{l_{1} t} \equiv$ $\tau\left(l_{1}+1, l_{2}, t+1, n\right)$ and so on. If we shift $l_{1} \rightarrow l_{1}+1$ in (3.2), we have

$$
\left(a_{2}-b\right) \tau_{l_{1} l_{2} t} \tau_{l_{1} n}+(b-c) \tau_{l_{1} l_{2}} \tau_{l_{1} t n}+\left(c-a_{2}\right) \tau_{l_{1} l_{2} n} \tau_{l_{1} t}=0
$$

Then, by imposing the reduction condition:

$$
\tau_{l_{1} l_{2}}=\tau
$$

the equation (3.3) turns to

$$
\left(a_{2}-b\right) \tau_{t} \tau_{l_{1} n}+(b-c) \tau \tau_{l_{1} t n}+\left(c-a_{2}\right) \tau_{n} \tau_{l_{1} t}=0 .
$$

Hence, putting $f:=\tau, g:=\tau_{l_{1}}$, we obtain

$$
\begin{aligned}
& \left(a_{1}-b\right) g_{t} f_{n}+(b-c) g f_{t n}+\left(c-a_{1}\right) g_{n} f_{t}=0, \\
& \left(a_{2}-b\right) f_{t} g_{n}+(b-c) f g_{t n}+\left(c-a_{2}\right) f_{n} g_{t}=0,
\end{aligned}
$$


and

$$
\frac{f g_{t n}}{g f_{t n}}=\frac{\left(a_{2}-b\right) f_{t} g_{n}+\left(c-a_{2}\right) f_{n} g_{t}}{\left(a_{1}-b\right) g_{t} f_{n}+\left(c-a_{1}\right) g_{n} f_{t}}=\frac{\left(c-a_{2}\right)+\left(a_{2}-b\right) \frac{f_{t} g_{n}}{f_{n} g_{t}}}{\left(a_{1}-b\right)+\left(c-a_{1}\right) \frac{g_{n} f_{t}}{g_{t} f_{n}}} .
$$

Now we denote

$$
x_{n}^{t}:=\frac{f g_{n}}{g f_{n}}, \quad y_{n}^{t}:=\frac{g f_{t}}{f g_{t}} .
$$

From the equality

$$
x_{n}^{t+1} y_{n+1}^{t}=x_{n}^{t} y_{n}^{t}=\frac{f_{t} g_{n}}{f_{n} g_{t}}, \quad \frac{x_{n}^{t+1}}{y_{n}^{t}}=\frac{f g_{t n}}{g f_{t n}},
$$

if we define $\alpha:=\frac{c-a_{1}}{c-b}, \beta:=\frac{a_{2}-b}{c-b}$, we find that $x_{n}^{t}, y_{n}^{t}$ defined in (3.5) satisfy the equation (2.1).

The $N$-soliton solution to (3.1) and (3.2) is known as

$$
\tau=\operatorname{det}_{1 \leq i, j \leq N}\left[\delta_{i j}+\frac{\gamma_{i}}{p_{i}-q_{j}}\left(\frac{q_{i}-a_{1}}{p_{i}-a_{1}}\right)^{l_{1}}\left(\frac{q_{i}-a_{2}}{p_{i}-a_{2}}\right)^{l_{2}}\left(\frac{q_{i}-b}{p_{i}-b}\right)^{t}\left(\frac{q_{i}-c}{p_{i}-c}\right)^{n}\right],
$$

where $\left\{p_{i}, q_{i}\right\}_{i=1}^{N}$ are distinct parameters from each other and $\left\{\gamma_{i}\right\}_{i=1}^{N}$ are arbitrary parameters [3]. The reduction condition (3.4) gives the constraint,

$$
\left(\frac{a_{1}-p_{i}}{a_{1}-q_{i}}\right)\left(\frac{a_{2}-p_{i}}{a_{2}-q_{i}}\right)=1
$$

to the parameters $\left\{p_{i}, q_{i}\right\}$. Since $p_{i} \neq q_{i}$, the constraint becomes $p_{i}+q_{i}=a_{1}+a_{2}$. By putting $\frac{p_{i}-a_{1}}{c-b} \rightarrow p_{i}, \frac{\gamma_{i}}{c-b} \rightarrow \gamma_{i}, \Delta:=\frac{a_{1}-a_{2}}{c-b}$ and $l_{1}=l_{2}$ we have

$$
\begin{aligned}
& f=\operatorname{det}_{1 \leq i, j \leq N}\left[\delta_{i j}+\frac{\gamma_{i}}{p_{i}+p_{j}+\Delta}\left(\frac{-p_{i}+\beta}{p_{i}+1-\alpha}\right)^{t}\left(\frac{p_{i}+1-\beta}{-p_{i}+\alpha}\right)^{n}\right], \\
& g=\operatorname{det}_{1 \leq i, j \leq N}\left[\delta_{i j}+\frac{\gamma_{i}}{p_{i}+p_{j}+\Delta} \frac{-\Delta-p_{i}}{p_{i}}\left(\frac{-p_{i}+\beta}{p_{i}+1-\alpha}\right)^{t}\left(\frac{p_{i}+1-\beta}{-p_{i}+\alpha}\right)^{n}\right] .
\end{aligned}
$$

Thus we obtain the $N$-soliton solution of (2.1) by (3.5), (3.6) and (3.7) in the field $\mathbb{F}_{q}(\epsilon)$.

We now return to the method of previous section. By substituting $\alpha=n_{a}+\epsilon, \beta=n_{b}+\epsilon$ $\left(n_{a}, n_{b} \in \mathbb{F}_{q}\right)$, we can construct soliton solutions in $\mathbb{F}_{q}(\epsilon)$ for suitable values of $\left\{p_{i}, \gamma_{i}\right\}$ and $\Delta$. We denote the solutions defined in $\mathbb{P F}_{q}$ when we put $\epsilon=0$ as $\tilde{f}, \tilde{g}, \tilde{x}_{n}^{t}$ and $\tilde{y}_{n}^{t}$. Figs. 6 and 7 show $\tilde{x}_{n}^{t}$ for one and two soliton solutions for the generalized discrete KdV equation (2.1). Lastly, we discuss the periodicity of the soliton solutions over $\mathbb{P F}_{q}$. We have

$$
\tilde{f}(n+q-1, t)=\tilde{f}(n, t+q-1)=\tilde{f}(n, t), \quad \tilde{g}(n+q-1, t)=\tilde{g}(n, t+q-1)=\tilde{g}(n, t),
$$

for all $t, n \in \mathbb{Z}$ since we have $a^{q-1} \equiv 1$ for all $a \in \mathbb{F}_{q}^{\times}$. Thus the functions $\tilde{f}$ and $\tilde{g}$ have periods $q-1$ over $\mathbb{F}_{q}$. However we cannot conclude that $\tilde{x}_{n}^{t}$ and $\tilde{y}_{n}^{t}$ are also periodic with periods $q-1$. The values of $\tilde{x}_{n}^{t}$ may not be periodic when $\tilde{f}(n, t) \tilde{g}(n+1, t)=0$ and $\tilde{g}(n, t) \tilde{f}(n+1, t)=0$ (see (3.5)). First we write $f(n, t) g(n+1, t)$ and $g(n, t) f(n+1, t)$ as follows:

$$
f(n, t) g(n+1, t)=\epsilon^{l} k(\epsilon), \quad g(n, t) f(n+1, t)=\epsilon^{m} h(\epsilon),
$$



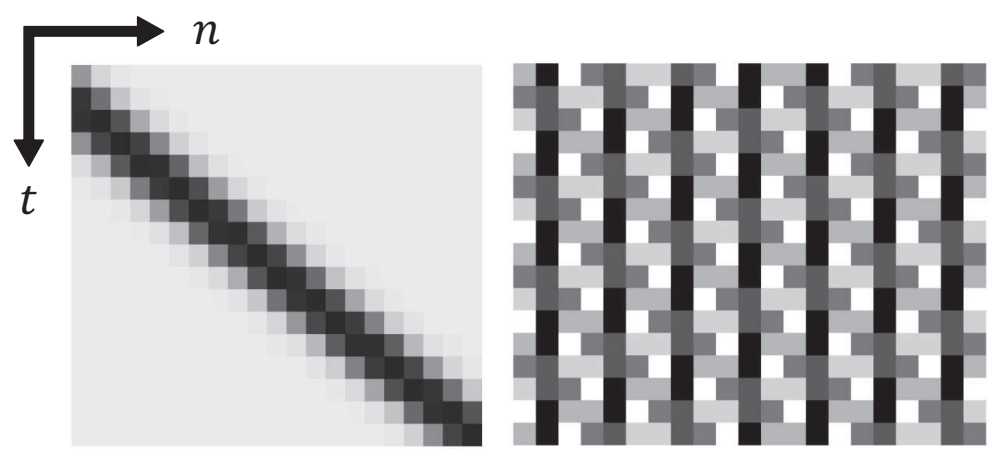

Figure 6. The one-soliton solution of the generalized discrete $\mathrm{KdV}$ equation (2.1) over $\mathbb{R}$ (left) and $\mathbb{P F}_{13}$ (right) where $\alpha=\frac{14}{15}, \beta=\frac{5}{6}, r_{1}=-\frac{1}{15}, l_{1}=\frac{1}{30}$. Elements of $\mathbb{P F}_{13}$ are represented on the following grayscale: from 0 (white) to 12 (gray) and $\infty$ (black).
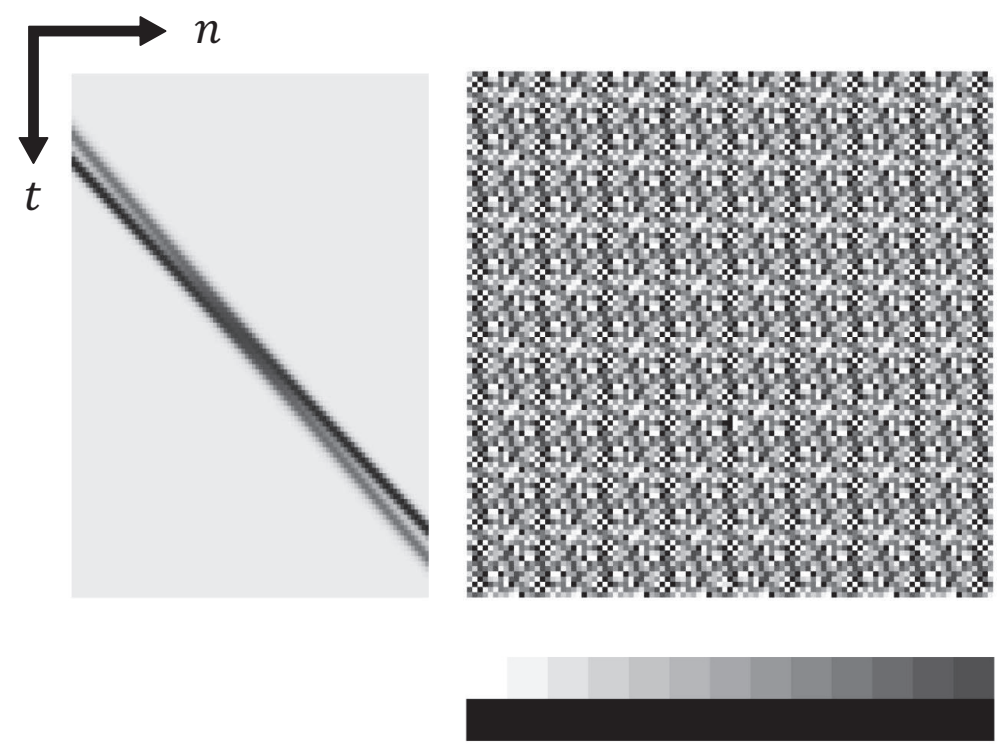

Figure 7. The two-soliton solution of the generalized discrete $\mathrm{KdV}$ equation (2.1) over $\mathbb{R}$ (left) and $\mathbb{P F}_{13}$ (right) where $\alpha=\frac{14}{15}, \beta=\frac{5}{6}, r_{1}=-\frac{1}{6}, l_{1}=\frac{2}{15}, r_{2}=-\frac{1}{30}, l_{2}=\frac{1}{30}$. Elements of $\mathbb{P F}_{13}$ are represented on the following grayscale: from 0 (white) to 12 (gray) and $\infty$ (black).

where $l, m \in \mathbb{Z}, h(0) \neq 0, k(0) \neq 0$ and $k(\epsilon), h(\epsilon) \in \mathbb{F}_{q}[\epsilon]$. We also write $f(n+q-1, t) g(n+q, t)=$ $\epsilon^{l^{\prime}} k^{\prime}(\epsilon), g(n+q-1, t) f(n+q, t)=\epsilon^{m^{\prime}} h^{\prime}(\epsilon)$ in the same manner. Let us write down the reduction map again:

$$
\tilde{x}_{n}^{t}= \begin{cases}\frac{k(0)}{h(0)}, & l=m, \\ 0, & l>m \\ \infty, & l<m .\end{cases}
$$

In the case when $\tilde{f}(n, t) \tilde{g}(n+1, t)=0$ and $\tilde{g}(n, t) \tilde{f}(n+1, t)=0, x_{n}^{t}=\frac{f(n, t) g(n+1, t)}{g(n, t) f(n+1, t)} \in \mathbb{F}_{q}(\epsilon)$ and $x_{n}^{t+q-1}=\frac{f(n+q-1, t) g(n+q, t)}{g(n+q-1, t) f(n+q, t)} \in \mathbb{F}_{q}(\epsilon)$ can have different reductions with respect to $\epsilon$, since $l^{\prime}$ is not necessarily equal to $l$, and neither $m^{\prime}$ is equal to $m$. The left part of Fig. 8 shows a magnified 


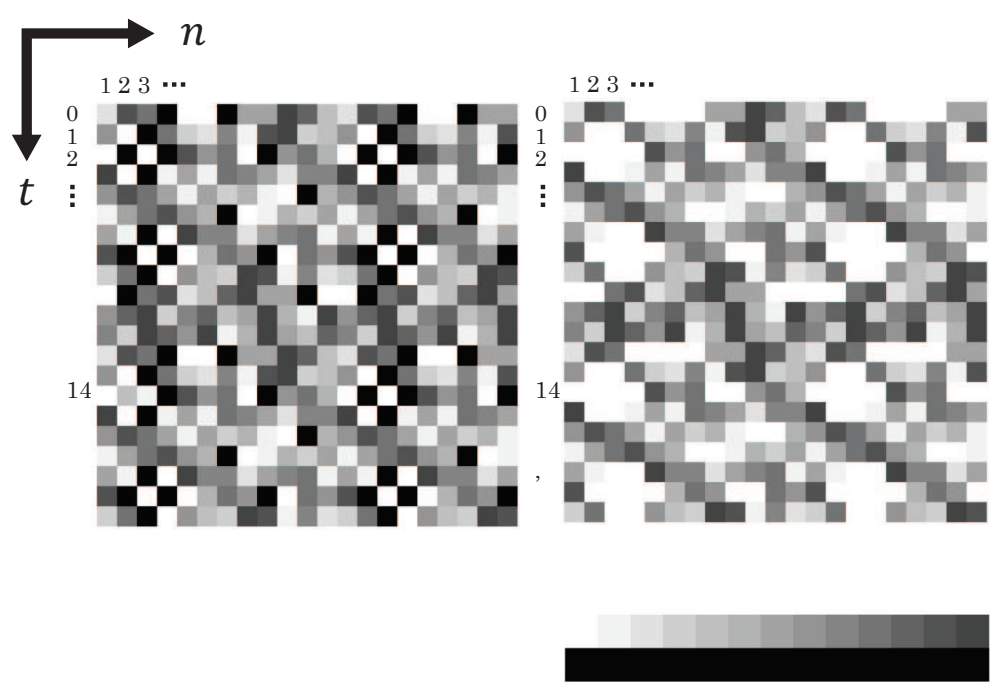

Figure 8. The two-soliton solution of the generalized discrete KdV equation (2.1) over $\mathbb{P F}_{13}$ calculated in two different ways. Elements of $\mathbb{P F}_{13}$ are represented on the following grayscale: from 0 (white) to 12 (gray) and $\infty$ (black).

plot of the same two-soliton solutions as in Fig. 7. In some points $\tilde{x}_{n}^{t}$ does not have period 12 (for example $x_{2}^{2} \neq x_{2}^{14}$ ) while almost all other points do have this periodicity.

If we want to recover full periodicity, there is another reduction to obtain $\tilde{x}_{n}^{t}$ and $\tilde{y}_{n}^{t}$ from $x_{n}^{t}$ and $y_{n}^{t}$. This time, we define $\tilde{x}_{n}^{t}$ as

$$
\tilde{x}_{n}^{t}= \begin{cases}\frac{k(0)}{h(0)}, & l=0, m=0, \\ 0, & \text { otherwise. }\end{cases}
$$

The right part of Fig. 8 shows the same two-soliton solution as in the left part but calculated with this new method. We see that all points have periods 12 . It is important to determine how to reduce values in $\mathbb{P F}_{q}(\epsilon)$ to values in $\mathbb{P F}_{q}$, depending on the properties one wishes the soliton solutions to possess.

\section{Discussion and concluding remarks}

We presented a prescription to obtain dynamical equations over finite fields from discrete equations defined over a field with characteristic 0 . The essential trick is to introduce an indeterminate (a parameter $\epsilon$ ) and regard the equations defined over $\mathbb{F}_{q}(\epsilon)$. By substituting $\epsilon$ with a value in $\mathbb{F}_{q}$, we can uniquely determine the values of the dependent variables in $\mathbb{P F}_{q}$. Furthermore if we have a one-parameter family of solutions to the original equation, we obtain the solution over a finite field straight away by substituting a suitable value for the parameter. The $N$-soliton solutions to the discrete $\mathrm{KdV}$ equation and the generalized discrete $\mathrm{KdV}$ equations over finite fields are thus obtained.

Our approach is also applied not only to the other discrete soliton equations but to ordinary nonlinear difference equations such as discrete Painlevé equations. Let us consider dP2 equation $[7,13]$ :

$$
x_{n+1}+x_{n-1}=\frac{z_{n} x_{n}+a}{x_{n}^{2}-1}, \quad n \in \mathbb{Z},
$$


where $z_{n}:=n \delta$ and $a, \delta$ are constants. If we examine (4.1) over $\mathbb{F}_{q}$, we cannot define its time evolution after the dependent variable $x_{n}$ takes \pm 1 . However, by regarding $a$ as an indeterminate, we can define the time evolution over $\mathbb{F}_{q}(a)$ and obtain a value in $\mathbb{P F}_{q}$ by substituting a value in $\mathbb{F}_{q}$ for $a$. The effectiveness of this approach is confirmed for wide range of ordinary discrete equations such as the other discrete or $q$-discrete Painlevé equations and the QRT mappings [16].

There is, however, another choice of indeterminate for the discrete ordinary difference equations. Let $\left(x_{0}, x_{1}\right)=(y, x)$ with $y \in \mathbb{F}_{q}$ in $(4.1)$. Then $x_{k}(k=2,3, \ldots)$ can be regarded as a function of $x$, i.e. $x_{k} \in \mathbb{F}_{q}(x)$. Since we have

$$
\begin{aligned}
& x_{2}=\frac{z_{1}+a}{2(x-1)}+\frac{\left(z_{1}-a\right)}{4}+O(x-1), \\
& x_{3}=-1+O(x-1), \\
& x_{4}=\frac{y\left(z_{1}+a\right)+2 a+\delta z_{2}}{a-z_{3}}+O(x-1), \\
& \ldots \ldots \ldots
\end{aligned}
$$

we can determine $x_{3}, x_{4}, \ldots$ by putting $x \rightarrow 1$ despite the fact that they are not well defined if we take $x_{1}=1$ in advance. The time evolution pattern thus obtained coincides with that of our approach with an indeterminate $a$.

The above procedure reminds us the singularity confinement method which is an effective test to judge the integrability of the given equations [5]. In fact, if we consider (4.1) over $\mathbb{C}$ and take $\epsilon:=x-1$ as an infinitesimal parameter, the time evolution pattern is exactly the one which passes the singularity confinement test. The grounds of this similarity become clear when one thinks of the theory of the space of initial conditions of the Painlevé equations [8, 9, 10, 11, 15]. As observed by Sakai [15], passing the singularity confinement test is essentially equivalent to the fact that the equation is lifted to an automorphism of the rational surface (the space of initial conditions) obtained by compactification and blowing-up from the original space of initial values $\mathbb{C} \times \mathbb{C}$. By introducing infinitesimal parameter in the case of singularity confinement test or an indeterminate in our approach, we avoid passing through a point on an exceptional curve generated by blowing-up and approximate the automorphism in an effective way. Hence these three types of approaches, that is, construction of space of initial conditions, application of singularity confinement and the method shown in the present paper, give the same time evolution rule for the discrete Painelevé equations over finite fields.

For soliton equations, however, we have difficulty in defining time evolution by constructing the space of initial conditions. Let us return to the equation (2.1). The mapping, $\left(x_{n}^{t}, y_{n}^{t}\right) \mapsto$ $\left(x_{n}^{t+1}, y_{n+1}^{t}\right)$, is lifted to an automorphism of the surface $\tilde{X}$, where $\tilde{X}$ is obtained from $\mathbb{P}^{1} \times \mathbb{P}^{1}$ by blowing up twice at $(0, \infty)$ and $(\infty, 0)$ respectively:

$$
\begin{aligned}
& \tilde{X}=\mathbb{A}_{1} \cup \mathbb{A}_{2}, \\
& \mathbb{A}_{1}:=\left\{\left(\left(x, \frac{1}{y}\right),[\xi: \eta],[u: v]\right) \mid x \eta-\frac{1}{y} \xi=0,\right. \\
&x((1-\alpha) \eta+\alpha \xi) v-((1-\beta) \eta+\beta \xi) u=0\} \subset \mathbb{A}^{2} \times \mathbb{P}^{1} \times \mathbb{P}^{1}, \\
& \mathbb{A}_{2}:=\left\{\left(\left(\frac{1}{x}, y\right),[\xi: \eta],[w: z]\right) \mid \frac{1}{x} \xi-y \eta=0,\right. \\
&y((1-\beta) \eta+\beta \xi) w-((1-\alpha) \eta+\alpha \xi) z=0\} \subset \mathbb{A}^{2} \times \mathbb{P}^{1} \times \mathbb{P}^{1},
\end{aligned}
$$

where $[a: b]$ denotes a set of homogeneous coordinates for $\mathbb{P}^{1}$. But, to define the time evolution 
of the system with $N$ lattice points from (2.1), we have to consider the mapping

$$
\left(y_{1}^{t} ; x_{1}^{t}, x_{2}^{t}, \ldots, x_{N}^{t}\right) \longmapsto\left(x_{1}^{t+1}, x_{2}^{t+1}, \ldots, x_{N}^{t+1} ; y_{N+1}^{t}\right) .
$$

Since there seems no reasonable decomposition of $\tilde{X}$ into a direct product of two independent spaces, successive use of (2.1) becomes impossible. Note that if we blow down $\tilde{X}$ to $\mathbb{P}^{1} \times \mathbb{P}^{1}$, the information of the initial values is lost in general. If we intend to construct an automorphism of a space of initial conditions, it will be inevitable to start from $\mathbb{P}^{N+1}$ and blow-up to some huge manifold, which is beyond the scope of the present paper. This difficulty seems to be one of the reasons why the singularity confinement method has not been used for construction of integrable partial difference equations or judgement for their integrability, though some attempts have been proposed in the bilinear form [14]. There should be so many exceptional hyperplanes in the space of initial conditions if it does exist, and it is practically impossible to check all the "singular" patterns in the naïve extension of the singularity confinement test. On the other hand, when we fix the initial condition for a partial difference equation, the number of singular patterns is restricted in general and we have only to enlarge the domain so that the mapping becomes well defined. This is the strategy that we adopted in this article.

As shown in the above discussion, the discrete Painlevé equations over finite fields can be treated by several methods. The comparison of the mathematical structure in $\mathbb{C}$ with that in $\mathbb{F}_{q}$ is one of the future problems. Clarifying the geometric and/or algebraic meaning of our approach to soliton equations and applications of our approach to the initial value problems related to curves over finite fields are also the problems we shall address in the future.

\section{Acknowledgement}

The authors wish to thank Professors K.M. Tamizhmani, R. Willox and Dr. S. Iwao for useful comments. This work is partially supported by Grant-in-Aid for JSPS Fellows (24-1379).

\section{References}

[1] Białecki M., Doliwa A., Discrete Kadomtsev-Petviashvili and Korteweg-de Vries equations over finite fields, Theoret. and Math. Phys. 137 (2003), 1412-1418, nlin.SI/0302064.

[2] Białecki M., Nimmo J.J.C., On pattern structures of the $N$-soliton solution of the discrete KP equation over a finite field, J. Phys. A: Math. Theor. 40 (2007), 949-959, nlin.SI/0608041.

[3] Date E., Jinbo M., Miwa T., Method for generating discrete soliton equation. II, J. Phys. Soc. Japan 51 (1982), 4125-4131.

[4] Doliwa A., Białecki M., Klimczewski P., The Hirota equation over finite fields: algebro-geometric approach and multisoliton solutions, J. Phys. A: Math. Gen. 36 (2003), 4827-4839, nlin.SI/0211043.

[5] Grammaticos B., Ramani A., Papageorgiou V., Do integrable mappings have the Painlevé property?, Phys. Rev. Lett. 67 (1991), 1825-1828.

[6] Kakei S., Nimmo J.J.C., Willox R., Yang-Baxter maps and the discrete KP hierarchy, Glasg. Math. J. 51 (2009), 107-119.

[7] Nijhoff F.W., Papageorgiou V.G., Similarity reductions of integrable lattices and discrete analogues of the Painlevé II equation, Phys. Lett. A 153 (1991), 337-344.

[8] Okamoto K., Studies on the Painlevé equations. I. Sixth Painlevé equation PI, Ann. Mat. Pura Appl. (4) 146 (1987), 337-381.

[9] Okamoto K., Studies on the Painlevé equations. II. Fifth Painlevé equation P $P_{\mathrm{V}}$, Japan. J. Math. (N.S.) 13 (1987), 47-76.

[10] Okamoto K., Studies on the Painlevé equations. III. Second and fourth Painlevé equations, $P_{\mathrm{II}}$ and $P_{\mathrm{IV}}$, Math. Ann. 275 (1986), 221-255.

[11] Okamoto K., Studies on the Painlevé equations. IV. Third Painlevé equation PIII, Funkcial. Ekvac. 30 (1987), 305-332. 
[12] Papageorgiou V.G., Tongas A.G., Veselov A.P., Yang-Baxter maps and symmetries of integrable equations on quad-graphs, J. Math. Phys. 47 (2006), 083502, 16 pages, math.QA/0605206.

[13] Ramani A., Grammaticos B., Hietarinta J., Discrete versions of the Painlevé equations, Phys. Rev. Lett. 67 (1991), 1829-1832.

[14] Ramani A., Grammaticos B., Satsuma J., Integrability of multidimensional discrete systems, Phys. Lett. A 169 (1992), 323-328.

[15] Sakai H., Rational surfaces associated with affine root systems and geometry of the Painlevé equations, Comm. Math. Phys. 220 (2001), 165-229.

[16] Tamizhmani K.M., Private communication, 2012.

[17] Wolfram S., Statistical mechanics of cellular automata, Rev. Modern Phys. 55 (1983), 601-644. 